\title{
ON THE SPECTRAL PICTURE OF AN IRREDUCIBLE SUBNORMAL OPERATOR
}

\author{
PAUL MCGUIRE
}

(Communicated by John B. Conway)

\begin{abstract}
This paper extends the following result of R. F. Olin and J. E. Thomson: A compact subset $K$ of the plane is the spectrum of an irreducible subnormal operator if and only if $\mathscr{R}(K)$ has exactly one nontrivial Gleason part $G$ such that $K$ is the closure of $G$. The main result of this paper is that the only additional requirement needed for the pair $\left\{K, K_{e}\right\}$ to be the spectrum and essential spectrum, respectively, is that $K_{e}$ be a compact subset of $K$ which contains the boundary of $K$. Additionally, results are obtained on the question of which index values can be specified on the various components of the complement of $K_{e}$.
\end{abstract}

Introduction and a survey of the results. All Hilbert spaces in this paper are separable and over the complex field. For a Hilbert space $\mathscr{H}, \mathscr{B}(\mathscr{H})$ denotes the algebra of bounded linear operators on $\mathscr{H}$ and $\mathscr{B}_{0}(\mathscr{H})$ denotes the ideal of compact operators on $\mathscr{H}$. If $A \in \mathscr{B}(\mathscr{H})$, then $\sigma(A)$ is the spectrum of $A$ and $\sigma_{e}(A)$ is the essential spectrum of $A$; that is $\sigma_{e}(A)$ is the spectrum of $A+\mathscr{B}_{0}(\mathscr{H})$ in $\mathscr{B}(\mathscr{H}) / \mathscr{B}_{0}(\mathscr{H})$. If $\lambda \notin \sigma_{e}(A)$, then $A-\lambda$ is a Fredholm operator and ind $(A-\lambda)=$ $\operatorname{dim} \operatorname{ker}(A-\lambda)-\operatorname{dim} \operatorname{ker}(A-\lambda)^{*}$ is the index. See Douglas [5] for properties of the index. In particular, $\operatorname{ind}(A-\lambda)$ is constant on components of $\mathbf{C} \backslash \sigma_{e}(A)$.

An operator $S$ on a Hilbert space $\mathscr{H}$ is subnormal if there is a normal operator $N$ on a Hilbert space $\mathscr{K}$ containing $\mathscr{H}$ such that $\mathscr{H}$ is invariant for $N$ and $S$ is the restriction of $N$ to $\mathscr{H}$. Amongst all such normal extensions of $S$, there exists a minimal normal extension which is unique up to a unitary equivalence. This minimal normal extension of $S$ will be denoted throughout this paper by $N$ or mne $(S)$. The operator $S$ is pure if there is no reducing subspace for $S$ on which $S$ is normal. It is well known that if $S$ is a pure subnormal operator, then ind $(S-\lambda) \leq-1$ for all $\lambda \in \sigma(S) \backslash \sigma_{e}(S)$.

If $K$ is a compact subset of the plane, then $\mathscr{C}(K)$ denotes the space of continuous functions on $K$ and $\mathscr{R}(K)$ the uniform closure in $\mathscr{C}(K)$ of the rational functions with poles off $K$. A Borel probability measure $\mu$ is a representing measure for $\mathscr{R}(K)$ at the point $a$ in $K$ if $f(a)=\int f d \mu$ for every $f$ in $\mathscr{R}(K)$. Two points $a$ and $b$ in $K$ belong to the same Gleason part of $\mathscr{R}(K)$ if there exists a constant $c>0$ such that $1 / c \leq u(a) / u(b) \leq c$ whenever $u$ is the real part of a function $f$ in $\mathscr{R}(K)$ and $u>0$. A nontrivial Gleason part is a part which contains at least two distinct points. A fundamental result concerning $\mathscr{R}(K)$ is that if $a$ and $b$ belong to the same Gleason part and $\mu_{a}$ is a representing measure for $\mathscr{R}(K)$ at $a$, then there exists a representing measure $\mu_{b}$ for $\mathscr{R}(K)$ at $b$ such that $\mu_{a}$ is absolutely

Received by the editors July 13, 1987 and, in revised form, December 29, 1987.

1980 Mathematics Subject Classification (1985 Revision). Primary 47B20; Secondary 47A10. 
continuous with respect to $\mu_{b}$ and the support of $\mu_{b}$ is contained in the boundary of $K$ together with the support of $\mu_{a}$. For these and other facts concerning $\mathscr{R}(K)$ see Conway [3] or Gamelin [7].

If $\mu$ is a positive, regular, Borel measure supported on $K$, then $\mathscr{R}^{2}(K, \mu)$ is the closure of $\mathscr{R}(K)$ in the Hilbert space $L^{2}(\mu)$. If $N_{\mu}$ is the operator on $L^{2}(\mu)$ given by $\left(N_{\mu} f\right)(z)=z f(z)$, then $\mathscr{R}^{2}(K, \mu)$ is invariant for $N_{\mu}$ and $S_{\mu}=\left.N_{\mu}\right|_{\mathscr{R}} ^{2}(K, \mu)$ is a rationally cyclic subnormal operator.

If $G$ is an open subset of $\mathbf{C}$, then $L_{a}^{2}(G)$ consists of those analytic functions $f: G \rightarrow \mathbf{C}$ such that

$$
\iint_{G}|f(z)|^{2} d \operatorname{Area}(z)<\infty .
$$

When endowed with the norm given by

$$
\|f\|_{L_{a}^{2}(G)}=\left(\iint_{G}|f|^{2} d \text { Area }\right)^{1 / 2},
$$

$L_{a}^{2}(G)$ is a Hilbert space. For general properties of subnormal operators, rationally cyclic subnormal operators, and $L_{a}^{2}(G)$, the reader is referred to Conway [3].

If $G$ is an open region in $\mathbf{C}$, then $H^{2}(G)$ consists of those analytic functions $f: G \rightarrow \mathbf{C}$ such that $|f|^{2}$ has a harmonic majorant. That is, there is a harmonic function $h: G \rightarrow[0, \infty)$ such that $|f(z)|^{2} \leq h(z)$ for all $z$ in $G$. Amongst all such harmonic majorants, there is a least harmonic majorant $h_{f}$. If $a$ is any fixed point in $G$, then $\|f\|=h_{f}(a)^{1 / 2}$. The space $H^{2}(G)$ is a Hilbert space when endowed with this norm. For general properties of $H^{2}(G)$ and its relationship to harmonic measure for $G$ evaluated at $a$, the reader is referred to Fisher [6] or Conway [4].

Throughout this paper the notation $\bar{K}$, int $(K)$, and $\partial K$ denotes the closure, interior, and boundary respectively of the set $K \subset \mathbf{C}$.

In Clancey and Putnam [2], it is shown that a necessary and sufficient condition for a compact set $K$ to be the spectrum of a pure subnormal operator is that $\mathscr{R}(K \cap D) \neq \mathscr{C}(K \cap D)$ whenever $D$ is an open disk intersecting $K$ in a nonempty set. An analogous result for irreducible subnormal operators appears in Olin and Thomson [11]. Here it is shown that the compact set $K$ is the spectrum of an irreducible subnormal operator if and only if $\mathscr{R}(K)$ has exactly one nontrivial Gleason part $G$ and $K$ is the closure of $G$. A difficulty with the Clancey-Putnam and Olin-Thomson results is that it is not possible to prescribe, or even describe, the essential spectrum of the subnormal operator constructed with the given spectrum $K$. This difficulty is partially removed in Hastings [9]. Here it is shown that the pair $\left\{K, K_{e}\right\}$ of compact sets consists of the spectrum and essential spectrum, respectively, of a pure subnormal operator if and only if $K$ satisfies the ClanceyPutnam condition and $\partial K \subset K_{e} \subset K$.

In this paper, it is shown in Theorem 5 that the pair $\left\{K, K_{e}\right\}$ of compact sets consists of the spectrum and essential spectrum, respectively, of an irreducible subnormal operator if and only if $K$ satisfies the Olin-Thomson condition and $\partial K \subset K_{e} \subset K$. Additionally it is shown that values of the index less than or equal to -2 can be prescribed on the various components of $K \backslash K_{e}$. Under what conditions the index value -1 can be prescribed is left as an open question. 
The main results. The main tool that is used in the proof of Theorem 5 is Theorem 4.1 of McGuire [10]. For completeness this result is restated below as Lemma 1 and a brief sketch of the construction is indicated. Lemmas 2, 3, and 4 below are preliminary to Theorem 5 .

LEMMA 1. Let $1 \leq n \leq \infty$ and let $\left\{\mu_{j}\right\}_{j=0}^{n}$ be a collection of pairwise singular, positive, regular, Borel measures which are supported on a compact subset of $\mathbf{C}$. For each $j=0, \ldots, n$ assume that:

(a) $\mathscr{H}_{j}$ is a subspace of $L^{2}\left(\mu_{j}\right)$ which is invariant for the operator $N_{j}$ on $L^{2}\left(\mu_{j}\right)$ defined by $\left(N_{j} f\right)(z)=z f(z)$ and $\mathscr{H}_{j}$ contains a nonzero constant function;

(b) if $S_{j}$ is the restriction of $N_{j}$ to $\mathscr{H}_{j}$, then $S_{j}$ is an irreducible subnormal operator with $N_{j}=\operatorname{mne}\left(S_{j}\right)$;

(c) there exists a bounded linear operator $A_{j}: \mathscr{H}_{0} \rightarrow \mathscr{H}_{j}$ such that $A_{j} S_{0}=S_{j} A_{j}$ and the range of $A_{j}$ contains a nonzero constant function.

Then there exists an irreducible subnormal operator $S$ such that $S$ is similar to $\bigoplus_{j=0}^{n} S_{j}$

Sketch of the Construction. Assume $n<\infty$. For each $j=1, \ldots, n$, let $\Delta_{j}$ be a subset of the support of $\mu_{j}$ such that $\mu_{j}\left(\Delta_{j}\right)>0$ and $\mu_{j}\left(\mathbf{C} \backslash \Delta_{j}\right)>0$. Let $P_{\Delta_{j}}: L^{2}\left(\mu_{j}\right) \rightarrow L^{2}\left(\mu_{j}\right)$ be the projection given by $P_{\Delta_{j}} f=\chi_{\Delta_{j}} f$ where $f \in L^{2}\left(\mu_{j}\right)$ and $\chi_{\Delta_{j}}$ denotes the characteristic function of $\Delta_{j}$. The operator $S$ is given by multiplication by $z$ on the subspace $\mathscr{H}$ of $\bigoplus_{j=0}^{n} L^{2}\left(\mu_{j}\right)$ defined by

$$
\begin{aligned}
\mathscr{H}=\left\{\left(f, P_{\Delta_{1}} A_{1} f+g_{1}, P_{\Delta_{2}} A_{2} f+g_{2}, \ldots, P_{\Delta_{n}} A_{n} f+g_{n}\right):\right. & \\
& \left.\quad f \in \mathscr{H}_{0} \text { and } g_{j} \in \mathscr{H}_{j}, 1 \leq j \leq n\right\} .
\end{aligned}
$$

LEMMA 2. Let $D$ be an open disc centered at $\lambda$ of radius $R$, let $A_{n}$ be the annulus $\{z: R(1-1 / n)<|z-\lambda|<R(1-1 /(n+1))\}$, let $m$ be a fixed positive integer, and for each $j=1, \ldots, m$, let $G_{j}=\bigcup_{k=0}^{\infty} A_{j+k m}$. If $f$ is an analytic function on $D$ such that $f \in L_{a}^{2}\left(G_{j}\right)$, then $f \in L_{a}^{2}(D)$ and there is a constant $C$ independent of $f$ and $R$ such that $\|f\|_{L_{a}^{2}(D)} \leq C\|f\|_{L_{a}^{2}\left(G_{j}\right)}$.

ProOF. Since $f$ is analytic on $D, M_{r}(f)=\int_{0}^{2 \pi}\left|f\left(\lambda+r e^{i \theta}\right)\right|^{2} d \theta$ is an increasing function of $r$ on $[0, R)$. For each $n=1,2, \ldots$, let $M_{n}(f)$ and $m_{n}(f)$ denote the supremum and infimum respectively of $\left\{M_{r}(f): \lambda+r\right.$ is in $\left.A_{n}\right\}$. It is straightforward to verify that

$$
\operatorname{Area}\left(A_{n}\right)=\frac{\pi R^{2}\left(2 n^{2}-1\right)}{(n+1)^{2} n^{2}} \leq \frac{18}{7} \frac{\pi R^{2}\left[2(n+1)^{2}-1\right]}{(n+2)^{2}(n+1)^{2}}=\frac{18}{7} \operatorname{Area}\left(A_{n+1}\right)
$$

and that the constant $\frac{18}{7}$ is independent of $R$. Hence

$$
\begin{aligned}
\iint_{A_{n}}|f|^{2} d \text { Area } & \leq M_{n}(f) \operatorname{Area}\left(A_{n}\right) \\
& \leq \frac{18}{7} m_{n+1}(f) \operatorname{Area}\left(A_{n+1}\right) \leq \frac{18}{7} \iint_{A_{n+1}}|f|^{2} d \text { Area. }
\end{aligned}
$$


Thus, if $I_{n}=\iint_{A_{n}}|f|^{2} d$ Area, then

$$
\begin{aligned}
\iint_{D}|f|^{2} d \text { Area }= & \sum_{n=1}^{\infty} I_{n} \\
= & \left(I_{1}+I_{2}+\cdots+I_{j}\right)+\sum_{k=0}^{\infty}\left(I_{j+k m+1}+I_{j+k m+2}+\cdots+I_{j+(k+1) m}\right) \\
\leq & {\left[\left(\frac{18}{7}\right)^{j-1}+\cdots+\left(\frac{18}{7}\right)+1\right] I_{j} } \\
& +\sum_{k=1}^{\infty}\left[\left(\frac{18}{7}\right)^{m-1}+\cdots+\left(\frac{18}{7}\right)+1\right] I_{j+k m} \\
\leq & \sum_{k=0}^{\infty}\left(\left(\frac{18}{7}\right)^{m-1}+\left(\frac{18}{7}\right)^{m-2}+\cdots+1\right) I_{j+k m} \\
= & C \int_{G_{j}}|f|^{2} d \text { Area }
\end{aligned}
$$

where $C$ is the constant $1+\frac{18}{7}+\cdots+\left(\frac{18}{7}\right)^{m-1}$.

Let $G$ be a bounded open set in $\mathbf{C}$, let $K_{0}=\{z \in G$ : $\operatorname{distance}(z, \partial G) \geq 1\}$, and, for $n \geq 1$, let $K_{n}=\{z \in G: 1 /(n+1) \leq \operatorname{distance}(z, \partial G)<1 / n\}$. Note $G=\bigcup_{n=0}^{\infty} K_{n}$ and for each $n \geq 0, \bar{K}_{n}$ is a compact subset of $G$. It is well known (see Proposition 2.4 on page 54 of Fisher [6]) that for each $n \geq 0$ there is a constant $c_{n}>0$ such that $|f(z)| \leq c_{n}\|f\|_{H^{2}(G)}$ for all $f$ in $H^{2}(G)$ and $z$ in $\bar{K}_{n}$. Hence if $\chi_{K_{n}}$ denotes the characteristic function of $K_{n}$ and $w_{1}(z)=\sum_{n=0}^{\infty}\left(1 / c_{n}^{2}\right) \chi_{K_{n}}(z)$, then for each $f$ in $H^{2}(G)$,

$$
\begin{aligned}
\iint_{G}|f(z)|^{2} w_{1}(z) d \operatorname{Area}(z) & =\sum_{n=0}^{\infty} \iint_{K_{n}}|f(z)|^{2} \frac{1}{c_{n}^{2}} d \operatorname{Area}(z) \\
& \leq \sum_{n=0}^{\infty} \iint_{K_{n}}\|f\|_{H^{2}(G)}^{2} d \operatorname{Area}(z) \\
& =\|f\|_{H^{2}(G)}^{2} \operatorname{Area}(G) .
\end{aligned}
$$

Now let $\left\{D_{k}\right\}_{k=1}^{\infty}$ be a sequence of pairwise disjoint, closed discs in $G$ with Area $(G)=\sum_{k=1}^{\infty} \operatorname{Area}\left(D_{k}\right)$. If $\lambda_{k}$ and $r_{k}$ are the center and radius respectively of the disc $D_{k}$, then for each $k=1,2, \ldots$ and $n=1,2, \ldots$, let $A_{n, k}=\left\{z: r_{k}(1-1 / n)<\right.$ $\left.\left|z-\lambda_{k}\right|<r_{k}(1-1 /(n+1))\right\}$. Fix a positive integer $m$ and for each $j=1,2, \ldots, m$, let $G_{j}=\bigcup_{k=1}^{\infty}\left(\bigcup_{n=0}^{\infty} A_{j+n m, k}\right)$. Note that for each disc $D_{k}, G_{j}$ contains every $m$ th annulus starting with the $j$ th annulus. Let $w_{2}(z)=\exp (-1 /$ distance $(z, \partial G))$ for each $z$ in $G$ and let $\mu_{j}$ be the measure given by $d \mu_{j}(z)=w_{1}(z) w_{2}(z) \chi_{G_{j}}(z) d \operatorname{Area}(z)$, where $\chi_{G_{j}}$ denotes the characteristic function of $G_{j}$. Let $\mathscr{R}(G)=\{r: r$ is a rational function with poles off $G\}$. Note that if $\lambda \in \partial G$, then $(z-\lambda)^{n} \in \mathscr{R}(G)$ for each integer $n$. Also note that the weight functions $w_{1}$ and $w_{2}$ are defined so that $(z-\lambda)^{n} \in L^{2}\left(\mu_{j}\right)$ for each integer $n$. Hence $\mathscr{R}(G) \subset L^{2}\left(\mu_{j}\right)$. Let $\mathscr{H}\left(G, \mu_{j}\right)$ be the 
closure of $\mathscr{R}(G)$ in $L^{2}\left(\mu_{j}\right)$ and let $S_{j}$ denote the operator on $\mathscr{H}\left(G, \mu_{j}\right)$ given by $\left(S_{j} f\right)(z)=z f(z)$.

LEMMA 3. If $S_{j}$ is the operator on $\mathscr{H}\left(G, \mu_{j}\right)$ described above, then $S_{j}$ is a subnormal operator such that $\sigma\left(S_{j}\right)=\operatorname{cl}(G), \sigma_{e}\left(S_{j}\right)=\partial G$, and $\operatorname{index}\left(S_{j}-\lambda\right)=-1$ for $\lambda$ in $G$. Moreover, if $G$ is connected, then $S_{j}$ is irreducible.

PROOF. It is clear that $S_{j}$ is a subnormal operator with minimal normal extension $N_{j}$ on $L^{2}\left(\mu_{j}\right)$ given by $\left(N_{j} f\right)(z)=z f(z)$.

If $\lambda \in \partial G$, then $\lambda$ is in the support of $\mu_{j}$. Hence $\lambda \in \sigma\left(S_{j}\right)$. Also if $\lambda \in \partial G$, then since $(z-\lambda)^{-n} \in \mathscr{R}(G)$ for each integer $n, \mathscr{R}(G)$ is contained in the range of $S_{j}-\lambda$. Since $\mathscr{R}(G)$ is dense in $\mathscr{H}\left(G, \mu_{j}\right)$ and $\lambda \in \sigma\left(S_{j}\right)$, it follows that $\lambda \in \sigma_{e}\left(S_{j}\right)$. Thus $\partial G \subset \sigma_{e}\left(S_{j}\right)$.

Now assume $\lambda \in G$ and let $D$ be a closed disc of radius $R$ centered at $\lambda$ and contained in $G$. Since $D$ is closed and contained in $G, \delta=\operatorname{distance}(D, \partial G)>0$. Let $\mathscr{F}=\left\{k: D_{k} \cap D\right.$ is not empty $\}$. Since each disc $D_{k}$ is closed and contained in $G, \delta_{k}=\operatorname{distance}\left(D_{k}, \partial G\right)>0$ for all $k=1,2, \ldots$ Since at most finitely many of the discs $\left\{D_{k}\right\}_{k=1}^{\infty}$ have diameter greater than $\delta / 2$, the distance from $\operatorname{cl}\left(\bigcup_{k \in \mathscr{F}} D_{k}\right)$ to $\partial G$ is positive. Hence area measure and $\mu_{j}$ are boundedly mutually absolutely continuous when restricted to $G_{j} \cap\left(\bigcup_{k \in \mathscr{F}} D_{k}\right)$. Thus by Lemma 2 , if $f \in \mathscr{H}\left(G, \mu_{j}\right)$, then $f \in L_{a}^{2}\left(\bigcup_{k \in \mathscr{F}} \operatorname{int}\left(D_{k}\right)\right)$. Since Area $(G)=\operatorname{Area}\left(\bigcup_{k=1}^{\infty} D_{k}\right)$, $\operatorname{Area}\left(D \backslash \bigcup_{k \in \mathscr{F}} D_{k}\right)=0$. Thus $f \in L_{a}^{2}(D)$ and

$$
|f(\lambda)|=\left|\frac{1}{2 \pi} \int_{0}^{2 \pi} f\left(\lambda+r e^{i \theta}\right) d \theta\right| \leq \frac{1}{2 \pi} \int_{0}^{2 \pi}\left|f\left(\lambda+r e^{i \theta}\right)\right| d \theta .
$$

Hence

$$
\begin{aligned}
|f(\lambda)| & \leq \frac{1}{\pi R^{2}} \int_{0}^{R} \int_{0}^{2 \pi}\left|f\left(\lambda+r e^{i \theta}\right)\right| r d \theta d r \\
& \leq \frac{1}{\pi R^{2}}\left(\iint_{D}|f|^{2} d \text { Area }\right)^{1 / 2}\left(\iint_{D} 1 d \text { Area }\right)^{1 / 2}=\frac{1}{R \sqrt{\pi}}\|f\|_{L_{a}^{2}(D)} \\
& \leq \frac{1}{R \sqrt{\pi}}\|f\|_{L_{a}^{2}\left(\bigcup_{k \in \mathscr{G}} \operatorname{int}\left(D_{k}\right)\right)} \leq C\|f\|_{L_{a}^{2}\left(\bigcup_{k \in \mathcal{F}}\left(D_{k} \cap G_{j}\right)\right)},
\end{aligned}
$$

by Lemma 2. Thus there is a constant $C$ such that $|f(\lambda)| \leq C\left\|f \chi_{D}\right\|_{L^{2}\left(\mu_{j}\right)} \leq$ $C\|f\|_{\mathscr{H}\left(G, \mu_{j}\right)}$. Hence evaluation at $\lambda$ is a bounded linear functional on $\mathscr{H}\left(G, \mu_{j}\right)$. Thus $L=\left\{f \in \mathscr{H}\left(G, \mu_{j}\right): f(\lambda)=0\right\}$ is a closed subspace with codimension one in $\mathscr{H}\left(G, \mu_{j}\right)$. Note that the range of $S_{j}-\lambda$ is contained in $L$. Also if $f \in L$, then $f(z)=$ $(z-\lambda) g(z)$ where $g=f /(z-\lambda)$ is analytic on $G \backslash\{\lambda\}$ and $\lambda$ is a removable singularity for $g$. Since $f \in \mathscr{H}\left(G, \mu_{j}\right)$, there is a sequence $\left\{r_{n}\right\}$ of rational functions in $\mathscr{R}(G)$ which converges to $f$ in $\mathscr{H}\left(G, \mu_{j}\right)$. A standard limiting argument now shows that $\left\{\left(r_{n}(z)-r_{n}(\lambda)\right) /(z-\lambda)\right\}$ converges to $(f(z)-f(\lambda)) /(z-\lambda)=(f(z)) /(z-\lambda)=g(z)$. Hence $g \in \mathscr{H}\left(G, \mu_{j}\right), L=\operatorname{range}\left(S_{j}-\lambda\right), S_{j}-\lambda$ is Fredholm, and index $\left(S_{j}-\lambda\right)=-1$.

Let $P$ denote the orthogonal projection of $L^{2}\left(\mu_{j}\right)$ onto $\mathscr{H}\left(G, \mu_{j}\right)$ and suppose $R$ is a projection which commutes with $S_{j}$. It follows from a theorem of Bram (see Bram [1] or Proposition 11.6 on page 198 of Conway [3]) that there is a projection $Q$ in the commutant of the von Neumann algebra generated by $N_{j}$ such that $Q$ commutes with $P$ and $R$ is the restriction of $Q$ to $\mathscr{H}\left(G, \mu_{j}\right)$. Since $N_{j}$ is cyclic, 
the commutant of the von Neumann algebra generated by $N_{j}$ is equal to $\left\{M_{\varphi}: \varphi \in\right.$ $\left.L^{\infty}\left(\mu_{j}\right)\right\}$ where $M_{\varphi}$ denotes the multiplication operator $\left(M_{\varphi} f\right)(z)=\varphi(z) f(z)$. Hence, there is a characteristic function $\chi$ in $\mathscr{H}\left(G, \mu_{j}\right)$ such that $R f=\chi f$ for all $f$ in $\mathscr{H}\left(G, \mu_{j}\right)$. Since $\chi$ is analytic on $G$, it follows that if $G$ is connected, then $\chi$ is either identically zero or identically one. Thus $S_{j}$ is irreducible if $G_{j}$ is connected.

The following "folk" lemma is well known but does not seem to appear in the literature. Since it is used repeatedly in the proof of Theorem 5, a brief sketch of its proof is included for the sake of completeness.

LEMMA 4. If $G$ is a bounded open region, $K=\bar{G}$, and $\omega$ is harmonic measure for $G$ evaluated at the interior point $a$ in $G$, then $\mathscr{R}^{2}(K, \omega)$ is isometrically contained in $H^{2}(G)$.

ProOF. It suffices to show that if $f$ is analytic in a neighborhood of $K$,

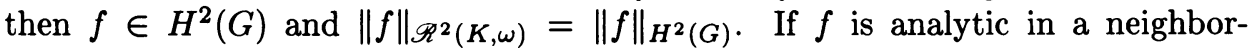
hood of $K$, then the Perron function $u_{f}$ associated with $|f|^{2}$ is a harmonic majorant of $|f|^{2}$ in $G$ and is given by $u_{f}(z)=\int|f(\varsigma)|^{2} d \omega_{z}(\zeta)$ where $\omega_{z}$ denotes harmonic measure for $G$ evaluated at $z$ (see part a of Proposition 7.4 on p. 330 of Conway [4]). Moreover, if $v$ is any other harmonic majorant of $|f|^{2}$, then $u_{f}(z)=\int|f(\varsigma)|^{2} d \omega_{z}(\varsigma) \leq \int v(\varsigma) d \omega_{z}(\varsigma)=v(z)$ since $f$ is analytic in a neighborhood of $K$. Also, $\|f\|_{H^{2}(G)}^{2}=u_{f}(a)=\int|f(\varsigma)|^{2} d \omega(\varsigma)=\|f\|_{\mathscr{R}^{2}(K, \omega)}^{2}$.

THEOREM 5. Let $K$ be a compact subset of $\mathbf{C}$ such that $\mathscr{R}(K)$ has exactly one nontrivial Gleason part $\Omega$ and $K=\bar{\Omega}$. If $K_{e}$ is a compact subset of $K$ such that $\partial K \subset K_{e}$, then there exists an irreducible subnormal operator $S$ with spectrum $K$ and essential spectrum $K_{e}$. Moreover if $V_{1}, V_{2}, \ldots$ are the components of $K \backslash K_{e}$ and $\left\{a_{n}\right\}_{n=1}^{\infty}$ is a sequence of integers such that $a_{n} \leq-2$ for all $n$, then $S$ may be chosen so that ind $(S-\lambda)=a_{n}$ for $\lambda \in V_{n}, n=1,2, \ldots$

PROOF. The first stage of the proof consists of the construction of an irreducible, rationally cyclic, subnormal operator $S_{\mu}$ such that $\sigma\left(S_{\mu}\right)=K, \sigma_{e}\left(S_{\mu}\right)=\partial K$, and $\operatorname{ind}\left(S_{\mu}-\lambda\right)=-1$ for $\lambda \in \operatorname{int}(K)$.

Let $\left\{\Omega_{j}\right\}_{j=1}^{n}$ denote the components of $\operatorname{int}(\Omega)$, the interior of $\Omega$. (If $\operatorname{int}(\Omega)$ is empty, then this step may be omitted.) For each $j=1,2, \ldots$, let $w_{j}$ denote a point in $\Omega_{j}$, and let $\omega_{j}$ denote harmonic measure for $\Omega_{j}$ evaluated at $w_{j}$. Let $z_{0}$ be a point in $\Omega$. Since $\omega_{j}$ is a representing measure for $\mathscr{R}(K)$ at the point $w_{j}$ and since $z_{0}$ and $w_{j}$ belong to the same Gleason part of $\mathscr{R}(K)$, there exists a representing measure $\eta_{j}$ for $\mathscr{R}(K)$ at $z_{0}$ such that $\omega_{j} \ll \eta_{j}$ and $\eta_{j}$ is supported on $\partial K$ (see Corollary 9.5 on page 348 of Conway [3] or Corollary 1.2 on page 143 of Gamelin [7]). If $n=\infty$, then let $\eta=\frac{1}{2} \sum_{j=1}^{n}\left(\eta_{j} / 2^{j}\right)$. If $n<\infty$, then let $\eta=(1 / 2 n) \sum_{j=1}^{n} \eta_{j}$. Since each $\eta_{j}$ is a probability measure, $\eta(\mathbf{C})=\frac{1}{2}$.

Let $\left\{z_{j}\right\}_{j=1}^{m}$ be a dense subset of $\Omega \backslash \operatorname{int}(\Omega)$. For each $z_{j}$, let $\nu_{j}$ be a representing measure for $\mathscr{R}(K)$ at $z_{0}$ such that $\nu_{j}$ is supported on $\left\{z_{j}\right\} \cup \partial K$ and $\delta_{j} \ll \nu_{j}$, where $\delta_{j}$ denotes the unit point mass measure at $z_{j}$. If $m<\infty$, then let $\nu=$ $(1 / 2 m) \sum_{j=1}^{m} \nu_{j}$. If $m=\infty$, then let $\nu=\frac{1}{2} \sum_{j=1}^{m}\left(\nu_{j} / 2^{j}\right)$. Since each $\nu_{j}$ is a probability measure, $\nu(\mathbf{C})=\frac{1}{2}$. Thus $\mu=\eta+\nu$ is a representing measure for $\mathscr{R}(K)$ at $z_{0}$. (If $\operatorname{int}(\Omega)$ is empty, then set $\mu=2 \nu$. If $\operatorname{int}(\Omega)=\Omega$, then set $\mu=2 \eta$.) 
Note that if $f \in \mathscr{R}^{2}(K, \mu)$, then $f \in \mathscr{R}^{2}\left(\operatorname{cl}\left(\Omega_{j}\right), \omega_{j}\right)$ for each $j=1, \ldots, n$. Hence $f \in H^{2}\left(\Omega_{j}\right)$ for each $j=1, \ldots, n$ by Lemma 4 . It is well known that if $T$ is the operator given by multiplication by $z$ on $H^{2}\left(\Omega_{j}\right)$, then $\Omega_{j} \subset \sigma(T) \backslash \sigma_{e}(T)$. Thus $\sigma_{e}\left(S_{\mu}\right) \subset \sigma_{e}(T) \subset \partial \Omega$ since $\mathscr{R}^{2}(K, \mu) \subset \mathscr{R}^{2}\left(\bar{\Omega}_{j}, \omega_{j}\right) \subset H^{2}\left(\Omega_{j}\right)$. Also $\partial K=\partial \Omega$ since $K=\bar{\Omega}$. Since $\partial K=\partial \Omega$ and the support of $\nu$ is $\Omega \backslash \operatorname{int}(\Omega)$, it follows that $\partial K \subset \sigma\left(S_{\mu}\right)$. Thus $\sigma\left(S_{\mu}\right)=K$ and $\sigma_{e}\left(S_{\mu}\right)=\partial K$. Since $S_{\mu}$ is rationally cyclic, $\operatorname{ind}\left(S_{\mu}-\lambda\right)=-1$ for all $\lambda \in \sigma\left(S_{\mu}\right) \backslash \sigma_{e}\left(S_{\mu}\right)$.

To show that $S_{\mu}$ is irreducible, let $R$ be a projection which commutes with $S_{\mu}$. Since mne $\left(S_{\mu}\right)$ is cyclic, it follows from Bram's theorem (see Proposition 11.6 on page 198 of Conway [3]) that $R=M_{\chi}$ where $\chi$ is a characteristic function in $\mathscr{R}^{2}(K, \mu)$ and $M_{\chi} f=\chi f$ for each $f \in \mathscr{R}^{2}(K, \mu)$. If $f \in \mathscr{R}(K)$, then $f\left(z_{0}\right)=$ $\int f d \mu=\langle f, 1\rangle$. Let $\left\{f_{n}\right\}$ be a sequence in $\mathscr{R}(K)$ which converges to $\chi$ in $L^{2}(\mu)$. Since $\left\langle f_{n}^{2}, 1\right\rangle=f_{n}\left(z_{0}\right) f_{n}\left(z_{0}\right)=\left\langle f_{n}, 1\right\rangle^{2}$ for each $n,\langle\chi, 1\rangle=\langle\chi, 1\rangle^{2}$. Thus $\langle\chi, 1\rangle=0$ or 1. Since $\langle\chi, 1\rangle=\int \chi d \mu$ and $\mu$ is a probability measure, it follows that $\chi$ is either 0 or 1 identically. Thus $R=0$ or 1 and $S_{\mu}$ is irreducible.

The next stage of the construction consists of producing a countable collection of subnormal operators which satisfies the hypothesis of Lemma 1 and which yields the prescribed spectral properties when Lemma 1 is applied. There are two steps in this stage. The first step involves arranging the essential spectrum and index values on the closure of $K \backslash K_{e}$. The second step involves placing the interior of $K_{e}$ in the essential spectrum.

Let $V_{k}$ be a component of $K \backslash K_{e}$. (If $K \backslash K_{e}$ is empty, then this step may be omitted.) Since $a_{k} \leq-2,\left|a_{k}\right|-1>0$. Let $S_{k, 1}, S_{k, 2}, \ldots, S_{k, m}$ be the irreducible, effectually rationally cyclic, subnormal operators obtained by applying Lemma 3 with $G=V_{k}$ and $m=\left|a_{k}\right|-1$. Hence $\sigma\left(S_{k, j}\right)=\bar{V}_{k}, \sigma_{e}\left(S_{k, j}\right)=\partial V_{k}$, and $\operatorname{ind}\left(S_{j, k}-\lambda\right)=-1$ for each $\lambda$ in $V_{k}$ and $j=1,2, \ldots,\left|a_{k}\right|-1$. Let $\mu_{k, j}$ be the measure corresponding to the measure $\mu_{j}$ in Lemma 3. Thus $S_{k, j}$ is the operator given by multiplication by $z$ on the space $\mathscr{H}\left(V_{k}, \mu_{k, j}\right)$ and $\left\{\mu_{k, j}\right\}_{j=1}^{m}$ is a collection of pairwise singular measures satisfying for each $j=1, \ldots,\left|a_{k}\right|-1$ :

(1) $\int|f|^{2} d \mu_{k, j} \leq \operatorname{Area}\left(V_{k}\right)\|f\|_{H^{2}\left(V_{k}\right)}^{2}$ for all $f \in H^{2}\left(V_{k}\right)$; and

(2) $\mu_{k, j}$ is singular with respect to $\mu$.

Note that inequality (1) above follows from the way in which the weight functions $w_{1}$ and $w_{2}$ were defined just prior to the statement of Lemma 3. Also note that since $V_{k}$ is a component of $K \backslash K_{e}, V_{k} \subset \Omega_{i}$ for some $i$. Thus by Lemma $4, \mathscr{R}^{2}(K, \mu) \subset \mathscr{R}^{2}\left(\bar{\Omega}_{i}, \omega_{i}\right) \subset H^{2}\left(\Omega_{i}\right) \subset H^{2}\left(V_{k}\right) \subset \mathscr{H}\left(V_{k}, \mu_{k, j}\right)$ and the operator $A_{k, j}: \mathscr{R}^{2}(K, \mu) \rightarrow \mathscr{H}\left(V_{k}, \mu_{k, j}\right)$ defined by $A_{k, j} f=f$ is a bounded linear operator whose range contains the constants. Also $A_{k, j} S_{\mu}=S_{k, j} A_{k, j}$ for each $k, j$, and $T_{1}=S_{\mu} \oplus\left(\bigoplus_{k, j} S_{k, j}\right)$ satisfies $\sigma\left(T_{1}\right)=K, \sigma_{e}\left(T_{1}\right)=\partial\left(K_{e}\right)$, and for each $k$, $\operatorname{ind}\left(T_{1}-\lambda\right)=a_{k}$ for $\lambda \in V_{k}$.

Let $\left\{B_{k}\right\}_{k=1}^{\infty}$ be a collection of pairwise disjoint open discs contained in $\operatorname{int}\left(K_{e}\right)$ such that $\overline{\operatorname{int}\left(K_{e}\right)}=\overline{\bigcup_{k=1}^{\infty} B_{k}}$. (If $\operatorname{int}\left(K_{e}\right)$ is empty, then omit this step.) If $r_{k}$ is the radius of the disc $B_{k}$, then let $\left\{r_{k, j}\right\}_{j=1}^{\infty}$ be an enumeration of the rational numbers in the interval $\left(0, r_{k}\right)$ and let $B_{k, j}$ be the disc of radius $r_{k, j}$ which is concentric with $B_{k}$. Let $S_{k, j}^{\prime}$ be the operator given by multiplication by $z$ on $H^{2}\left(B_{k, j}\right)$ and let $\mu_{k, j}^{\prime}$ denote arclength measure on $\partial B_{k, j}$. Since $\operatorname{int}\left(K_{e}\right) \subset \Omega$, each $B_{k}$ is contained in a component of $\Omega$. By Lemma 4 , the operator $A_{k, j}^{\prime}: \mathscr{R}^{2}(K, \mu) \rightarrow H^{2}\left(B_{k, j}\right)$ defined 
by $A_{k, j}^{\prime} f=f$ is a bounded linear operator whose range contains the constants and $A_{k, j}^{\prime} S_{\mu}=S_{k, j}^{\prime} A_{k, j}^{\prime}$. Also note that $\{\mu\} \cup\left\{\mu_{k, j}\right\}_{k, j} \cup\left\{\mu_{k, j}^{\prime}\right\}_{k, j}$ is a collection of pairwise singular measures, and that $S_{k, j}^{\prime}$ is an irreducible, cyclic, subnormal operator with $\sigma\left(S_{k, j}^{\prime}\right)=\overline{B_{k, j}}, \sigma_{e}\left(S_{k, j}^{\prime}\right)=\partial B_{k, j}$, and $\operatorname{ind}\left(S_{k, j}^{\prime}-\lambda\right)=-1$ for $\lambda \epsilon$ $B_{k, j}$. Hence $T_{2}=\bigoplus_{k, j} S_{k, j}^{\prime}$ is a subnormal operator with spectrum and essential spectrum equal to $\overline{\operatorname{int}\left(K_{e}\right)}$.

The final stage in the construction is to apply Lemma 1 to the countable collection of operators $S_{\mu} \cup\left\{S_{k, j}\right\}_{k, j} \cup\left\{S_{k, j}^{\prime}\right\}_{k, j}$ with $\mu_{0}=\mu, S_{0}=S_{\mu}$, and $\mathscr{H}_{0}=$ $\mathscr{R}^{2}(K, \mu)$. The irreducible subnormal operator $S$ obtained is similar to $T_{1} \oplus T_{2}$ and thus has the desired spectral properties.

The first stage of the proof of Theorem 5 is of independent interest and is stated below as Corollary 6.

COROLlARY 6. Let $K$ be a compact subset of $\mathbf{C}$ such that $\mathscr{R}(K)$ has exactly one nontrivial Gleason part $\Omega$ and $K=\bar{\Omega}$. If $z_{0}$ is a point in $G$, then there is a representing measure $\mu$ for $\mathscr{R}(K)$ at the point $z_{0}$ such that the rationally cyclic subnormal operator $S_{\mu}$ on $\mathscr{R}^{2}(K, \mu)$ satisfies $\sigma\left(S_{\mu}\right)=K$ and $\sigma_{e}\left(S_{\mu}\right)=\partial K$.

It is also worth remarking that if in Theorem 5 it is assumed that $\operatorname{Area}\left(K_{e}\right)=0$ and $\sum_{j} a_{j} \operatorname{Area}\left(V_{j}\right)<\infty$, then the operator $S$ has the additional property that $S^{*} S-S S^{*}$ is a trace class operator (see Corollary 9 of Hadwin and Nordgren [8]).

\section{BIBLIOGRAPHY}

1. J. Bram, Subnormal operators, Duke Math. J. 22 (1955), 75-94.

2. K. Clancey and C. R. Putnam, The local spectral behavior of completely subnormal operators, Trans. Amer. Math. Soc. 163 (1972), 239-244.

3. J. B. Conway, Subnormal operators, Research Notes in Math., vol. 51, Pitman, London, 1981.

4. J. B. Conway, Spectral properties of certain operators on Hardy spaces of planar regions, preprint.

5. R. G. Douglas, Banach algebra techniques in operator theory, Academic Press, New York, 1972.

6. S. Fisher, Function theory on planar domains, Wiley, New York, 1983.

7. T. W. Gamelin, Uniform algebras, Chelsea, New York, 1986.

8. D. Hadwin and E. Nordgren, The Berger-Shaw Theorem, Proc. Amer. Math. Soc. (to appear).

9. W. W. Hastings, The approximate point spectrum of a subnormal operator, J. Operator Theory 5 (1981), 119-126.

10. P. J. McGuire, On $C^{*}$-algebras generated by a subnormal operator, preprint; J. Functional Anal. 79 (1988).

11. R. F. Olin and J. E. Thomson, Irreducible operators whose spectra are spectral sets, Pacific J. Math. 91 (1980), 431-434.

Department of Mathematics, BuCKNell University, LeWisburg, PenNSylvaNIA 17837 